\title{
ARQ Optimization for UMTS Networks
}

\author{
${ }^{1}$ Eduardo Rodrigues Vale, ${ }^{2}$ Julio Cesar R. Dal Bello \\ ${ }^{1,2}$ (Telecommunications Department, School of Engineering / Federal Fluminense University, Brazil)
}

\begin{abstract}
Initially developed for use in fixed networks, the ARQ (Automatic Repeat Request) technique was adopted in UMTS systems aiming the QoS improvement for data transmission. However, real time services like VoIP cannot benefits of ARQ, because of the related delay problems in WCDMA systems. With the advent of HSPA (High Speed Packet Access) and the LTE (Long Term Evolution) networks, there was a consequent redefinition of the ARQ functionalities, aiming to become possible to use it for real time services. Delay problems were minimized as a consequence of the facilities introduced for the new methods of ARQ generation. The main objective of this work is the definition of a methodology toward ARQ optimization relatively to the mean power required to transfer the information from the origin to the destination, accordingly to a given error probability.To reduce the amount of the power transfer in the uplink direction is of paramount importance for the minimization of interference levels as well as to economize battery of cellular equipment.Though the treatment here presented is focused to E-DCH (Enhanced Uplink Dedicated Channel) of HSPA systems, the results obtained are applicable to other related networks using ARQ techniques.
\end{abstract}

Keywords: Umts, Arq, Wcdma, Lte, Hspa

\section{Introduction}

The ARQ technology proves to be very useful for data transmission in WCDMA systems. Because this process is executed at RLC (Radio Link Control) level, the resultant delay does not permit its utilization for real time services. This situation has changed with the introduction of HSPA systems, where some ARQ functionalities were transfered to the Physical level (PHY). The main consequence was a delay reduction in the information transfer, thus allowing the ARQ utilization also for real time services.

In any case, it is important to maintain low levels of power transmission, in order to economize cellular batteries as well as to reduce interference levels. Here we present a methodology for optimization of ARQ power transmission, with the definition of an algorithm for the immediate calculation of the power levels of signal retransmissions in order to attend a given BER (Bit Error Rate) specified for the service. The emphasis is the treatment of VoIP transmission at E-DCH, though the conceps here presented are useful for other ARQ applications.

Some works have already considered this subject but to the best of the author's knowledge, the approach here developed has not yet been addressed in the technical literature. The paper is organized as follows: after the Introduction, Section II describes the theory behind ARQ optimization; Section III shows the theory for the determination of the Error Function; Section IV describes the algorithm for implementation of ARQ optimization; Finally, Section V presents the conclusions.

\section{Theory For Arq Optimization}

The study of the ARQ process requires the definition of a model, to obtain the necessary knowledge of the contribution of the different parts involved. Figure 1 presents a simplified view of a UMTS transmission system (Physical Level)[1] emphasizing the components that have direct influence in ARQ.

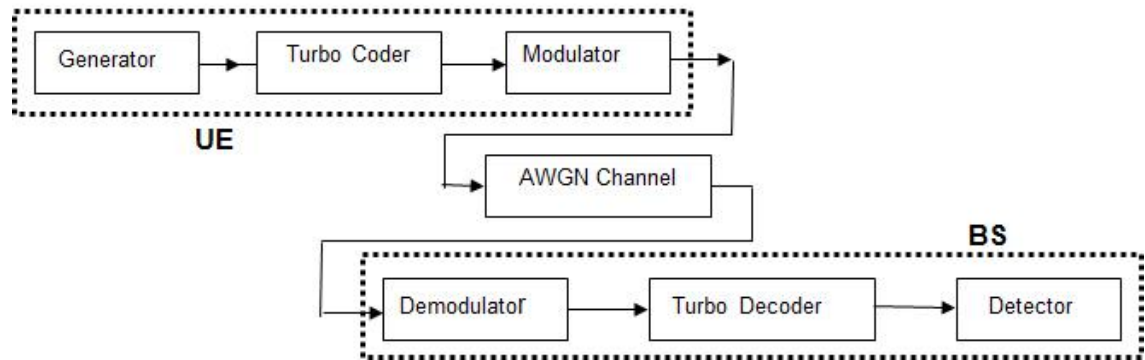

Figure 1 - Transmission System

Considering the uplink direction, the Generator is capable of sending information packets with length of $N$ bits. The Turbo Coder operates with a rate of 1:3, consequently generating an output of $3 x N$ bits. It follows 
the Modulator (BPSK) block. These components are located in the user cellular equipment (UE). Here it is considered a white gaussian noise channel (AWGN block). After the demodulation of the signal, the Turbo Decoder associated with the Detector block (both situated in the base station - BS) tries to recover the original signal.

For a given service, there is a BER (Bit Error Rate) value considered as the maximum acceptable. Therefore, to comply with this specified BER value we have two possibilities, in UMTS systems: to increase the transmission power and/or to allow the repetition of the packet transmission in case of a detected error in the previous transmission. We will be considering the chase combining principle adopted by the 3GPP (Third Generation Partnership Project) for the UMTS, where all the packet contents are retransmitted. It is easy to see that if we have more than one chance to transmit the same packet, we can use lower levels of power in order to achive a desired BER level, compared with the case of one unique transmission. Then the problem is to find the minimum power level[2],[3],[4] associated with each transmission attempt for the achievement of a given BER level associated with a specified service. This is the main objective of this paper. It will be considered the situations of one to $n$ repetitions (two to $n+1$ transmissions in total). As a result of this optimization for the power transmission levels we can obtain two imediate consequences for the cellular system: it will be possible to economize battery of the user cellular equipment and the interference levels of the system will be reduced, with the consequent reduction of noise levels, thus increasing the capacity of the cellular system[5].

Considering a transmission system specified for $n$ repetitions, it is possible to define the following events:

- $A_{l}$ - The packet is correctly detected in the first transmission;

- $A_{2}$ - The packet is correctly detected in the second transmission;

- $A_{n}$ - The packet is correctly detected in the nth transmission;

Then, defining as $P(A)$ the probability to receive the packet correctly we have:

$$
P(A)=P\left(A_{1}\right)+P\left(A_{2} \cap \overline{A_{1}}\right)+P\left(A_{3} \cap \overline{A_{1}} \cap \overline{A_{2}}\right)+\ldots+P\left(A_{n} \cap \overline{A_{1}} \cap \overline{A_{2}} \cap \overline{A_{3}} \cap \ldots \cap \overline{A_{n-1}}\right)
$$

Considering the natural independence of the events, and after the following substitutions in the previous equation,

$$
P\left(A_{1}\right)=\left[1-P\left(\overline{A_{1}}\right)\right] ; P\left(A_{2}\right)=\left[1-P\left(\overline{A_{2}}\right)\right] ; \ldots . P\left(A_{n}\right)=\left[1-P\left(\overline{A_{n}}\right)\right]
$$

we have, after some transformations:

$$
P(A)=1-P\left(\overline{A_{1}}\right) \cdot P\left(\overline{A_{2}}\right) \cdot P\left(\overline{A_{3}}\right) \ldots P\left(\overline{A_{n}}\right)
$$

Figure 2 shows an hiphotetical curve of $\mathrm{BER} \times \mathrm{Eb} / \mathrm{No}(\mathrm{dB})$ where $\mathrm{Eb}$ is the energy per bit of the signal and No is the termal noise density at the reception.

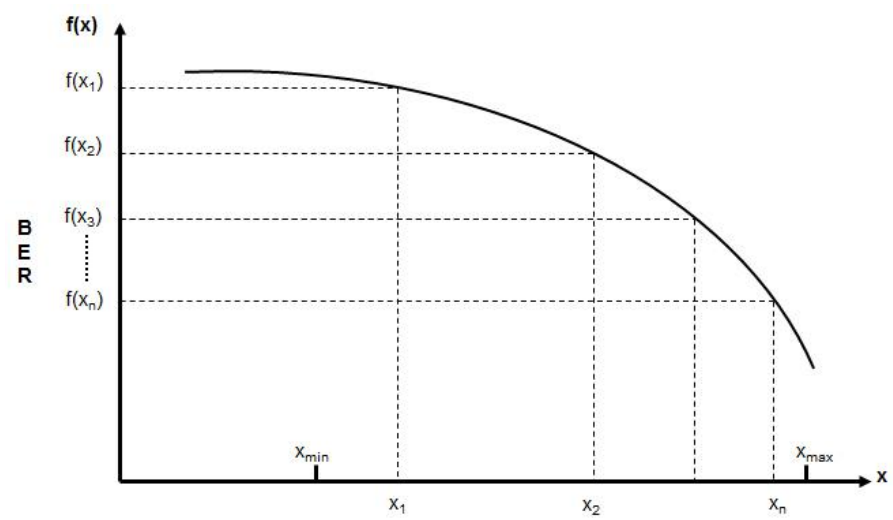

Figure 2 - BER x Eb/No(dB)

In order to simplify the expressions, consider the following change of variables: 


$$
P\left(\overline{A_{1}}\right)=f\left(x_{1}\right) ; \quad P\left(\overline{A_{2}}\right)=f\left(x_{2}\right) ; \quad P\left(\overline{A_{3}}\right)=f\left(x_{3}\right) ; \ldots . \quad P\left(\overline{A_{n}}\right)=f\left(x_{n}\right)
$$

Equation (1) can be rewritten as:

$$
P(A)=1-f\left(x_{1}\right) \cdot f\left(x_{2}\right) \cdot f\left(x_{3}\right) \ldots f\left(x_{n}\right)
$$

In the figure, $x_{1}, x_{2}, \ldots, x_{n}$ represent the different power levels (in $\mathrm{dB}$ ) assumed for each transmission and $f\left(x_{1}\right), f\left(x_{2}\right), \ldots, f\left(x_{n}\right)$ are the corresponding BER values. The points $\mathbf{x}_{\min }, \mathbf{x}_{\max }$ define the interval of interest. We have the following possibilities, for the amount of power dispended in the process of transfering one packet correctly:

- If the packet is transfered correctly in the first transmission, the power dispended is $x_{l}$. The associated probability of ocurrence is $P\left(A_{1}\right)$;

- If the packet is transfered correctly in the second transmission, the power dispended is $x_{1+} x_{2}$. The associated probability of ocurrence is $P\left(\overline{A_{1}}\right)$;

- This process continues until the packet is received correctly or the maximum number of retransmissions $(n)$ is reached;

Table 1, summarises these possibilities:

Table 1 - Power and Probability of Occurrence

\begin{tabular}{|c|c|c|}
\hline Number of Transmissions & Power Dispended & Probability of Occurrence \\
\hline 1 & $\mathrm{x}_{1}$ & $P\left(A_{l}\right)$ \\
\hline 2 & $\mathrm{x}_{2}$ & $P\left(\overline{A_{1}}\right)$ \\
\hline$\ldots$ & $\ldots$ & $\ldots$ \\
\hline $\mathrm{n}$ & $\mathrm{x}_{1}+\mathrm{x}_{2}+\ldots+\mathrm{x}_{\mathrm{n}}$ & $P\left(\overline{A_{1}}\right) \times P\left(\overline{A_{2}}\right) \times \ldots \times P\left(\overline{A_{n}}\right)$ \\
\hline
\end{tabular}

Using (2) and the results of Table 1 we can write the expression for the average power comsumption of an ARQ process with a maximum of $n-1$ retransmissions:

$$
\begin{aligned}
& C_{m}=x_{1}\left[1-f\left(x_{1}\right)\right]+\left(x_{1}+x_{2}\right) \cdot f\left(x_{1}\right)+\left(x_{1}+x_{2}+x_{3}\right) \cdot f\left(x_{1}\right) f\left(x_{2}\right)+\ldots+\left(x_{1}+x_{2}+\ldots+x_{n}\right) \cdot f\left(x_{1}\right) \cdot f\left(x_{2}\right) \ldots f\left(x_{n-1}\right) \\
& \text { or, } \\
& C_{m}=x_{1}+x_{2} \cdot f\left(x_{1}\right)+\left(x_{1}+x_{2}+x_{3}\right) \cdot f\left(x_{1}\right) f\left(x_{2}\right)+\ldots+\left(x_{1}+x_{2}+\ldots+x_{n}\right) \cdot f\left(x_{1}\right) \cdot f\left(x_{2}\right) \ldots f\left(x_{n-1}\right)
\end{aligned}
$$

Now it is important to to make some considerations about $f(x)$. This is the objective of next section.

\section{Determination Of The Error Function F(X)}

In fact, $f$ is a function of two variables: the Eb/No quantity and the packet lenght $\mathrm{N}$. Due to the intrinsic caractheristics of the expressions it is more convenient to solve this problem using simulation procedures. For this purpose it was developed a Matlab program based on Simulink, with the blocks defined by Figure 1. The results are showed by the collection of points appearing in Figure 2, representing the variation of BER x Eb/No for three packet length $(\mathrm{N})$ values in bits.

A visual inspection in the data generated by the simulation has revealed that a suitable approximation function is of the form:

$$
f\left(N, E_{b} / N_{0}\right)=10^{\sum_{i=0}^{n_{1}} a_{i}(N) \cdot\left(E_{b} / N_{0}\right)^{i}}
$$

where $n_{1}$ was chosen to be 6 and $E b / N o$ is expressed in $\mathrm{dB}$.

Each one of the $a$ 's coefficients can be estimated in the LS sense and they can be further modelled by an expression of the form:

$$
a_{i}(N)=\sum_{j=0}^{n_{2}} b_{i j} \cdot N^{j}
$$

where $n_{2}$ was chosen to be 2 .

Therefore the probability in question was modelled by: 


$$
P\left(N, E_{b} / N_{0}\right)=10^{g\left(N, E_{b} / N_{0}\right)}
$$

where

$$
g\left(N, E_{b} / N_{0}\right)=\sum_{i=0}^{n_{1}} \sum_{j=0}^{n_{2}} b_{i j} \cdot\left(E_{b} / N_{0}\right)^{i} \cdot N^{j}
$$

The curves of Figure 2 represent the results of this model when applied to the rate 1/3 turbo code used in the UMTS system. From there we can notice a fairly reasonable adjustment between the model and the empirical data obtained by long run simulation.

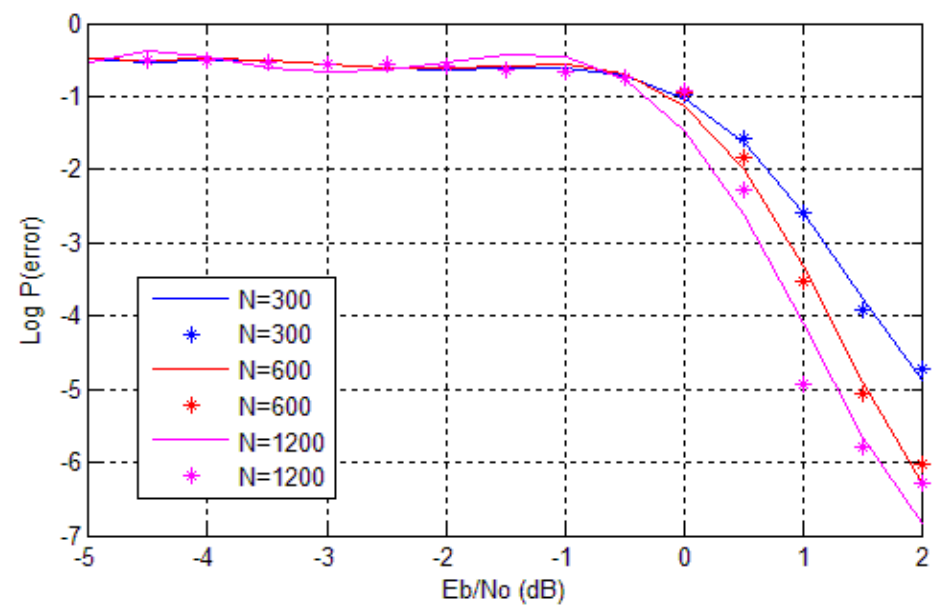

Figure 2: BER x Eb/No Curves

\section{Algorithm For Cm Minimization}

The results of the last section allow us to define an algorithm for the determination of $C_{m}$ and the respectives $x_{1}, \ldots, x_{n}$ values, for a given $\mathrm{P}(\mathrm{A})$. The steps of the algorithm are the following:

1. Do

$$
x_{1}=x_{2}=x_{3}=\ldots=x_{n-1}=x_{\min }
$$

2. Considering the analytical expression of $f(x)$ (expression (8)) it is straightforward to obtain:

$$
f\left(x_{1}\right)=f\left(x_{2}\right)=f\left(x_{3}\right)=\ldots=f\left(x_{n-1}\right)
$$

3. With the knowledge of $n$ and $P(A)$, expression (3) gives:

$$
f\left(x_{n}\right)-\frac{1-P(A)}{f\left(x_{1}\right) \cdot f\left(x_{2}\right) \ldots . f\left(x_{n-1}\right)}=0
$$

4. Using the Bisection Method we can determine the root of equation (9), consequently giving us the $x_{n}$ value.

5. Taking the values obtained in steps 1,2 and 4 in expression (4) we obtain the $C_{m}$ value.

6. Return to step 1 with:

$$
\begin{aligned}
& x_{1}=x_{2}=x_{3}=\ldots=x_{n-2}=x_{\min } \\
& x_{n-1}=x_{n-1}+\Delta
\end{aligned}
$$

7. Step 6 will be repeated until $x_{n-1}=x_{\max }$. Afterwards, the same procedure will be applied for $x_{n-2}$ and then successively until $x_{I}=x_{\max }$.

8. We select the least of the $C_{m}$ values obtained from all the above interactions. This step ends the algorithm. 


\section{Conclusions}

This work showed a technique, implemented by the construction of an algorithm, which is able to minimize the energy consumption for the operation of ARQ[6]. This technique, although developed for the purpose of saving the battery life of mobile cellular devices may be employed in other types of networks that utilize ARQ. In a future work, which is being finalized by the authors, it is presented a practical application of the theory described here.

Finally, it is important to note that the algorithm analyzed is easy to implement and requires minimal computational resources for operation.

\section{References}

[1]. 3GPP Document, Radio Link Control (RLC) Protocol Specification, TS 25.322, v6.0.0, December 2012.

[2]. Sohn I. and Bang S.C., Performance Studies of Rate Matching for WCDMA Mobile Receiver, IEEE VTC 2000, 52nd, vol.6, pp.2661-2665, 2000 .

[3]. Aftelak S. and Bhatoolaul, Rate Matching Attribute Settings and Error Rate Performance Sensitivity for Selected UMTS FDD Services, IEEE Semiannual Vehicular Technology Conference Final Program, October 6-9, 2003.

[4]. Vale E., Brandão J.C., Grivet M., UMTS Rate Matching Optimization, International Association of Science and Technology for Development - IASTED, CSA 2005, Banff, Canada, July 2005.

[5]. Hammer F., Reichl P., Nordstrom T., Kubin G., Corrupted Speech Data Considered Useful, Acta Acustica, Vol.90, No. 6, pp 10521060, Nov/Dec 2004.

[6]. Baey S., Dumas M. and Dumas M. C., QoS Tuning and Resource Sharing for UMTS WCDMA Multiservice Mobile, IEEE Transactions on Mobile Computing, vol.1, No. 3, pp. 221-235, July-Sept. 2002. 